\title{
Does Hurthle Cell Lesion/Neoplasm Predict Malignancy More Than Follicular Lesion/Neoplasm on Thyroid Fine-Needle Aspiration?
}

\author{
Robert T. Pu, M.D., Ph.D., ${ }^{1 *}$ Jack Yang, M.D., ${ }^{2}$ Patricia G. Wasserman, M.D., ${ }^{2}$ \\ Tawfiqul Bhuiya, M.D., ${ }^{2}$ Kent A. Griffith, M.P.H., M.S., ${ }^{3}$ and Claire W. Michael, M.D. ${ }^{1}$
}

Thyroid fine-needle aspiration (FNA) is a standard procedure for the clinical triage of thyroid nodules. The diagnosis of an adequately sampled thyroid FNA is generally grouped into three categories: benign, malignant, and indeterminate. The latter group usually includes follicular neoplasm, follicular lesion, and sometimes a more specific diagnosis such as Hurthle cell neoplasm or follicular lesion/neoplasm with Hurthle cell change. Whether a FNA diagnosis of Hurthle cell lesion/neoplasm (HLN) denotes a worse clinical outcome than follicular lesion/neoplasm (FLN) remains controversial. A cohort of 303 thyroid FNA cases with follow-up thyroidectomy in our institutes was identified, with the follow-up excision diagnosis compared to the FNA diagnosis in order to address this issue. Of this cohort, 87 cases had an FNA diagnosis of HLN while 216 cases had a diagnosis of FLN. Upon excision, the FNA diagnosis of HLN group had 14 cases of goiter/nodular hyperplasia (16\%), 46 cases of adenoma (12 follicular adenoma (14\%) and 34 cases of Hurthle cell adenoma (39\%)), and 27 cases of carcinoma (31\%, 12 papillary carcinoma and 15 Hurthle cell carcinoma). The FLN group had 74 cases of goiter/nodular hyperplasia (34.3\%), 8 cases of Hashimoto thyroiditis (3.7\%), 73 cases of follicular adenoma (33.8\%), one case of granular cell tumor, and 60 cases of carcinoma $(27.8 \%, 46$ papillary carcinoma, 12 follicular carcinoma, and 1 Hurthle cell carcinoma and 1 parathyroid carcinoma) upon excision. There is no significant difference in predicting cancer between the two cytology diagnosis groups (HLN versus $F L N, 31 \%$ versus $27.8 \%, P=0.5771$ ). When sorting all the cases by the surgical diagnosis, while comparable for age at diagnosis, the cancer group having the higher proportion of male patients than the non-cancer group (28.7\% versus $16.7 \%$,

${ }^{1}$ Department of Pathology, Cancer Center, University of Michigan Medical School, Ann Arbor, Michigan

${ }^{2}$ Long island Jewish Medical Center, New Hyde Park, New York

${ }^{3}$ Biostatistic Unit, Cancer Center, University of Michigan Medical School, Ann Arbor, Michigan

Present address: J. Yang, Department of Pathology, University of Texas Medical Branch at Galveston

*Correspondence to: Robert T. Pu, M.D., Ph.D., Department of Pathology, University of Michigan Medical School, 1500 E. Medical Center Drive, Room 2G332/BOX 0054, Ann Arbor, MI.

E-mail: robertpu@umich.edu

Received 11 July 2005; Accepted 6 October 2005

DOI 10.1002/dc.20440

Published online in Wiley InterScience (www.interscience.wiley.com).
$P=0.0259)$. Hurthle cell carcinoma patients are typically older than patients with other cancer diagnoses (59 versus 44, $P=$ 0.0077). Our results suggest that an FNA diagnosis of HLN does not predict more malignancy than FLN. Males and older patients with a HLN FNA diagnosis carry a higher risk of Hurthle cell carcinoma upon thyroidectomy. Diagn. Cytopathol. 2006;34:330-334. ๑ 2006 Wiley-Liss, Inc.

Key Words: thyroid FNA; follicular lesion/neoplasm; Hurthle cell lesion/neoplasm

Thyroid fine-needle aspiration (FNA) is a standard triage procedure in the management of thyroid nodules. ${ }^{1-6}$ The FNA diagnosis of an adequately sampled thyroid nodule generally can be grouped into benign, malignant, and indeterminate categories. Satisfactory thyroid FNA samples generally contain follicular cells and colloid. While cellular, samples classified as indeterminate generally contain little colloid with follicular cells that possess worrisome features, such as microfollicles and cells in syncytial arrangements with the loss of the normal honeycomb sheet. In these cases, the definitive diagnosis of hyperplasia versus neoplasia or adenoma versus carcinoma cannot be made based on the accepted cytomorphologic criteria. The diagnosis for these cases is generally coined "follicular lesion/neoplasm." This general diagnosis can encompass cellular hyperplastic nodule, thyroiditis, adenoma, and other cancer types, which are often diagnosed as follicular carcinoma or follicular variants of papillary carcinoma upon histological examination of the subsequent thyroidectomy specimen. For yet other indeterminate FNA cases with predominance of Hurthle cells, the more specific diagnosis of Hurthle cell lesion/neoplasm (HLN) is often given. Although there is no consensus about the diagnostic guidelines for HLN, one group offered the following criteria: a cellular specimen with a monotonous population of oncocytic/Hurthle cells (in excess of $70 \%$ of the specimen), associated with scant or absent colloid 
and without the nuclear features of papillary thyroid carcinoma. $^{7}$

Hurthle cell carcinoma is a rare disease. When stratified by the stage of disease and degree of differentiation, the aggressiveness of Hurthle cell carcinoma is similar to follicular carcinoma. ${ }^{8}$ However, distinguishing Hurthle cell adenoma from carcinoma using only the cytology specimen is extremely difficult.

The clinical decision-making regarding the treatment of thyroid nodules with an indeterminate cytological diagnosis has historically depended upon the size of the nodules and the patient's age, among other risk factors. ${ }^{5,9-11}$ Given the aggressiveness of Hurthle cell carcinoma, clinicians, when faced with the diagnosis of HLN after FNA, normally opt to treat their patients with a more aggressive surgical approach. However, whether a diagnosis of HLN on FNA truly predicts a worse disease state than the more general diagnosis of follicular lesion/neoplasm (FLN) on FNA has not been adequately addressed. ${ }^{7}$ Some authors have reported that the diagnosis of HLN carries a much higher risk of malignancy determined at thyroidectomy, while others have not. ${ }^{712-14}$ Therefore, we have conducted this study to answer the following question: Does the diagnosis of HLN on FNA carry a worse prognosis (as measured by the pathologic assessment of the surgical excision specimen) than the diagnosis of FLN?

\section{Materials and Methods}

Thyroid FNA cases were identified by searching of files of the Departments of Pathology at University of Michigan from 1995 to 2003, and at the Long Island Jewish Medical Center from 1992 to 2002. The cases identified included FNAs performed by cytopathologists, radiologists, or endocrinologists. The adequacy of most specimens was evaluated by on-site cytopathologists. To determine the adequacy of the specimen, a drop from the FNA sample was placed on one slide and smeared with a second, resulting in two slides. One slide was then air-dried for the determination of adequacy by Diff-Qick staining, while the second slide was immediately fixed and later Papanicolaou stained. FNAs performed by clinicians without the on-site assessment of adequacy were rinsed in Cytolyt and then processed using the ThinPrep method for Papanicolaou staining. The adequacy of these specimens was based on the Papanicolaou Society of Cytopathology guideline published in $1996 .{ }^{15}$ All cases with an indeterminate cytologic diagnosis on FNA, including follicular lesion, follicular neoplasm, Hurthle cell lesion, or Hurthle cell neoplasm, who had thyroidectomy and surgical histopathologic diagnosis were identified. These cases were subdivided into HLN and FLN groups. The followup surgical histopathologic diagnoses were compared between these FNA groups. Demographic information was obtained from the patient's pathology report and included gender and age at diagnosis.

Student's $t$-test was used to test for differences in the mean age between groups, and Fisher's exact test was used to test for differences in the proportion in malignant diagnoses between groups.

\section{Results}

A total of 4,920 thyroid FNA cases were identified by searching the archives at the University of Michigan Department of Pathology from 1995 to 2003 and at the Long Island Jewish Medical Center from 1992 to 2002. Of these, the FNA diagnosis of $303(6 \%)$ cases was indeterminate and a follow-up surgical resection was performed. Among these, 216 (71\%) cases had a diagnosis of FLN while 87 (29\%) cases had the more specific diagnosis of either Hurthle cell neoplasm or follicular lesion/ neoplasm with Hurthle cell change. The follow-up surgical histopathologic diagnoses were grouped into cancer and noncancer groups. Noncancer cases included nodular hyperplasia, thyroiditis, follicular and Hurthle cell adenoma. Cancer cases included follicular carcinoma, Hurthle cell carcinoma, and papillary carcinoma. Diagnoses of papillary carcinoma did not include incidental papillary carcinoma that was less than $1 \mathrm{~cm}$ in diameter and having dominant nodule diagnosed as a benign process or adenoma. The demographic features of the patients were similar between the two FNA groups (HLN group versus HLN group) and are presented in Table I. The mean age was 50.3 versus 46.7 and the proportion of females was 82 versus $79 \%$, respectively.

Comparison of the surgical resection diagnoses between the two FNA groups revealed that 60 of the 216 patients $(27.8 \%)$ in the FLN group had a diagnosis of carcinoma, while 27 of $87(31 \%)$ of the patients in the HLN group had a diagnosis of carcinoma (Table I and Fig. 1). Although the proportion of malignant cases was slightly higher for the HLN group, it was not a statistically meaningful difference $(P=0.5771)$. The cancer diagnoses in the HLN group $(N=27)$ were comprised of papillary carcinoma $(N=12,44 \%)$ and Hurthle cell carcinoma $(N=$ $15,56 \%)$. The cancer diagnoses of the FLN group ( $N=$ $60)$, however, were comprised of predominantly papillary carcinoma $(N=46,77 \%)$, while follicular carcinoma made up 20\% $(N=12)$ (Table I). Together, more than half of the carcinoma diagnoses from the cohort were papillary carcinoma (58 of $87,67 \%$, Table I). Twelve cases of follicular carcinoma were among the 60 cases of carcinoma in the FLN group. No cases of follicular carcinoma were identified in the HLN group. However, one case of Hurthle cell carcinoma was found in the FLN group. Fifteen patients in the HLN group were found to have Hurthle cell carcinoma. Although only $1 / 3$ of the cases on surgical follow-up turned out to be carcinoma 
Table I. Age, Gender, and Cancer Subtype at Surgical Excision by FNA Cohort

\begin{tabular}{|c|c|c|}
\hline Characteristic & $\begin{array}{l}\text { Hurthle cell } \\
\text { lesion/neoplasm } \\
(N=87)\end{array}$ & $\begin{array}{c}\text { Follicular } \\
\text { lesion/neoplasm } \\
(N=216)\end{array}$ \\
\hline \multicolumn{3}{|l|}{ Age (yr) } \\
\hline Mean & 50.25 & 46.74 \\
\hline Standard error & 1.82 & 1.06 \\
\hline Range & $14-89$ & $16-83$ \\
\hline$P$-value & 0.0968 & \\
\hline \multicolumn{3}{|l|}{ Gender } \\
\hline Male, N (\%) & $16(18.4)$ & $45(20.8)$ \\
\hline$P$-value & 0.7518 & \\
\hline \multicolumn{3}{|l|}{ Surgical pathology subtype } \\
\hline Goiter/hyperplasia & $14(16.1)$ & $74(34.3)$ \\
\hline Follicular adenoma & $12(13.8)$ & $73(33.8)$ \\
\hline Hurthle cell adenoma & $34(39.1)$ & 0 \\
\hline Hashimoto's & 0 & $8(3.7)$ \\
\hline Granular cell tumor & 0 & $1(0.5)$ \\
\hline Papillary carcinoma & $12(13.8)$ & $46(21.3)$ \\
\hline Follicular carcinoma & 0 & $12(5.6)$ \\
\hline Hurthle cell carcinoma & $15(17.2)$ & $1(0.5)$ \\
\hline Parathyroid carcinoma & 0 & $1(0.5)$ \\
\hline \multicolumn{3}{|c|}{$\begin{array}{l}\text { Surgical pathology follow-up } \\
\text { cancer sub-type }\end{array}$} \\
\hline Cancer, $N(\%)$ & $27(31.0)$ & $60(27.8)$ \\
\hline$P$-value & 0.5771 & \\
\hline \multicolumn{3}{|l|}{ Age (yr) } \\
\hline Mean & 52.1 & 44.5 \\
\hline Standard error & 3.6 & 2.03 \\
\hline Range & $14-82$ & $17-82$ \\
\hline$P$-value & 0.0721 & \\
\hline \multicolumn{3}{|l|}{ Gender } \\
\hline Male, $N(\%)$ & $8(29.6)$ & $17(28.3)$ \\
\hline$P$-value & 0.9999 & \\
\hline
\end{tabular}

overall, the HLN FNA diagnosis was much more predictive of a definitive diagnosis of Hurthle cell carcinoma than the FLN FNA diagnosis was for a definitive diagnosis of follicular carcinoma ( $56 \%$ versus $20 \%, P=0.021)$.

When sorting the cases by their surgical excision diagnosis of cancer, the groups were comparable for age (46.8 versus $48.1, P=0.54$ ), but had a significantly higher proportion of male cancer patients (28.7\%) compared with noncancer patients $(16.7 \%, P=0.0259$, Table II). In addition, diagnoses of Hurthle cell carcinoma were made in significantly older patients (59 versus $44, P=0.0077$, Table III) than the other cancer types.

\section{Discussion}

By reviewing thyroid FNAs performed at our institutions over a period of 8-10 yr, we have found no significant difference between the two indeterminate thyroid FNA diagnoses, FLN versus HLN, in predicting malignancy on subsequent surgical resection. Although the HLN group had a higher percentage of patients with malignancy diagnosis on the follow-up surgical specimen (31.0\%) than that of the FLN group $(27.8 \%)$, the difference is not statistically significant. Other studies, although not designed

\section{Follicular Lesion/Neoplasm (216 cases)}

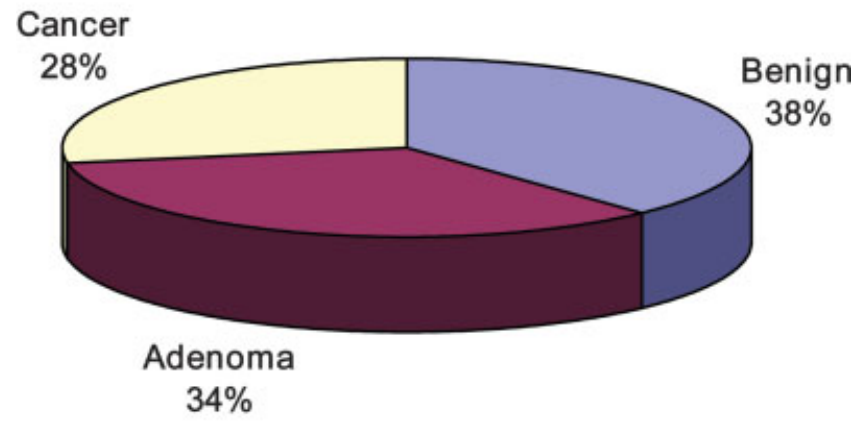

Hurthle Cell Lesion/Neoplasm (87 cases)

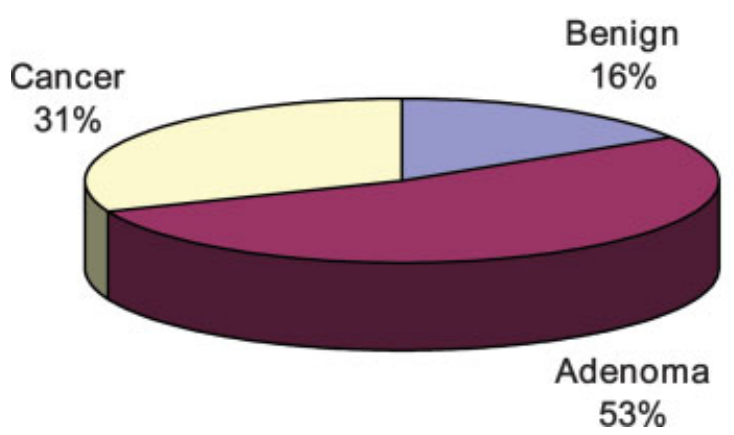

Fig. 1. Surgical outcomes of the two FNA subgroups. Top panel: Follicular lesion/neoplasm group has 82 benign, 74 adenoma, and 60 cancer cases on follow-up thyroidectomy. Bottom panel: Hurthle cell lesion/neoplasm group has 14 benign, 46 adenoma, and 27 cancer cases on followup thyroidectomy. [Color figure can be viewed in the online issue, which is available at www.interscience.wiley.com.]

to address this exact question, have reported similar findings, which suggest that a patient is not at a significantly higher risk of having malignant disease with an FNA diagnosis of HLN than FLN. ${ }^{12-14}$ However, one report did recently show that there was much higher risk of malignancy when the FNA diagnosis of "Hurthle cell neoplasm/follicular neoplasm with oncocytic features" was rendered. ${ }^{7}$ In that report by Giordadze et al., it was noted that the follow-up surgical diagnosis of malignancy was much higher $(45 \%)$ than what others, and we, have noted (about 30\%). One obvious reason for the difference is that our cohort included cases with FNA diagnoses of follicular and Hurthle cell lesion, in addition to cases with diagnoses of follicular neoplasm and Hurthle cell neoplasm. Thus, we expect our cohort to include more "benign" lesions, resulting in a lower rate of malignancy upon follow-up excision. Interestingly, the same group's earlier publication did not find a higher risk associated with follicular lesion/neoplasm with Hurthle cell change, 
Table II. Age and Gender by Cancer vs NonCancer Distinction on Surgical Excision (For All the Patients in the Cohort)

\begin{tabular}{lcc}
\hline Characteristic & Cancer $(N=87)$ & Noncancer $(N=216)$ \\
\hline Age $(\mathrm{yr})$ & & \\
Mean & 46.83 & 48.12 \\
Standard error & 1.83 & 1.06 \\
Range & $14-82$ & $14-89$ \\
$P$-value & 0.5428 & \\
Gender & & $36(16.7)$ \\
Male, $N(\%)$ & $25(28.7)$ & \\
$P$-value & 0.0259 & \\
\hline
\end{tabular}

although the sample size was too small for a valid statistical analysis. ${ }^{16}$ Their subsequent report, however, did report on the combined cases from two large institutions with well-known expertise in thyroid pathology in US and Italy. ${ }^{7}$ Underlying differences between our population and theirs may contribute to the difference observed. In addition, their report did not compare the two cytological diagnoses using the same patient population. Our data were collected at two large institutions, and the two groups of thyroid FNA diagnoses case were analyzed from the same time period using the same patient pool. However, our data relied upon the historical FNA diagnosis, which would be expected to have some degree of interobserver variability, especially during the time period prior to the adoption of the guidelines for thyroid FNA adequacy by Papanicolaou Society of Cytopathology. In contrast, the Giordadze group used a more specifically defined criteria for the Hurthle cell neoplasm group. ${ }^{7}$

We also noted that when a diagnosis of either FLN or HLN was rendered, the excisional surgical pathology diagnosis was of papillary carcinoma in about $2 / 3$ of the carcinoma cases (58 of 87 cases). This further demonstrated that the indeterminate nature of this cytological diagnosis as follicular variant of papillary carcinoma is one of the well-known differentials and is usually very difficult to distinguish from follicular neoplasm.

The aim of this study was not to identify clinical risk factors but to determine whether the cytological grouping of FLN versus HLN can be of use in predicting different outcome. To this end, we did not detect any noticeable difference. There are, however, more Hurthle cell carcinoma cases in the HLN group, although the overall rate of carcinoma is similar to that of the FLN group. As Hurthle cell carcinoma and follicular carcinoma have a similar and more aggressive nature when compared to papillary carcinoma, the FNA diagnosis of HLN does carry a more grave outcome (due to higher proportion of Hurthle cell carcinoma), but not in the rate of malignancy. Although we did not detect any differences in age and gender in the patients from the two cytological diagnosis groups, males did make up a higher proportion of the patients with a cancer diagnosis. Similarly, others have also shown that male gender is a risk factor for thyroid
Table III. Age and Gender of Hurthle Cell Carcinoma vs Other Cancer Diagnoses (For All the Cancer Patients in the Cohort)

\begin{tabular}{lcc}
\hline Characteristic & $\begin{array}{c}\text { Hurthle cell } \\
\text { carcinoma } \\
(N=16)\end{array}$ & $\begin{array}{c}\text { Other cancer } \\
\text { diagnosis } \\
(N=71)\end{array}$ \\
\hline Age $(\mathrm{yr})$ & 59.38 & \\
$\quad$ Mean & 4.85 & 44.00 \\
$\quad$ Standard error & $28-82$ & 1.80 \\
Range & 0.0077 & $14-77$ \\
$P$-value & $6(37.5)$ & \\
Gender & 0.3800 & $19(26.8)$ \\
$\quad$ Male, $N(\%)$ & & \\
$P$-value & & \\
\hline
\end{tabular}

cancer. ${ }^{16,17}$ The age of patients from the two groups was similar, but the subsequent cancer patients were older in the HLN group than in the FLN group, albeit not significantly. However, Hurthle cell carcinoma patients tended to be older than that of the other types of cancer (57 versus $44, P=0.007)$. So, older patients with a HLN diagnosis may be considered to have a higher risk of cancer.

In conclusion, we found that a diagnosis of HLN does not impart a higher rate of malignancy than a diagnosis of FLN in FNA of thyroid nodules. They predicted similar portions of carcinoma on subsequent thyroidectomy and they ought to be regarded similarly as a cytological indeterminate diagnosis group, although the ultimate patient outcome may be dictated by more Hurthle cell carcinomas found in HLN group in comparison to the FLN group. Factors such as male gender and older age are in general associated with worse outcome, especially with Hurthle cell carcinoma.

\section{References}

1. Ravetto C, Colombo L, Dottorini ME. Usefulness of fine-needle aspiration in the diagnosis of thyroid carcinoma: a retrospective study in 37,895 patients. Cancer 2000;90:357-363.

2. Bahar G, Braslavsky D, Shpitzer T, et al. The cytological and clinical value of the thyroid "follicular lesion". Am J Otolaryngol 2003; 24:217-220.

3. Blansfield JA, Sack MJ, Kukora JS. Recent experience with preoperative fine-needle aspiration biopsy of thyroid nodules in a community hospital. Arch Surg 2002;137:818-821.

4. Caplan RH, Strutt PJ, Kisken WA, et al. Fine-needle aspiration biopsy of thyroid nodules. Wis Med J 1991;90:285-288.

5. Fernandes JK, Day TA, Richardson MS, et al. Overview of the management of differentiated thyroid cancer. Curr Treat Options Oncol 2005;6:47-57.

6. Gelderblom AJ, vd Hoek W, Lips PT, et al. A study of the importance of fine-needle aspiration cytology in the diagnosis of the solitary thyroid nodule. Neth J Med 1990;36:13-18.

7. Giorgadze T, Rossi ED, Fadda G, et al. Does the fine-needle aspiration diagnosis of "Hurthle-cell neoplasm/follicular neoplasm with oncocytic features" denote increased risk of malignancy? Diagn Cytopathol 2004;31:307-312.

8. Evans HL, Vassilopoulou-Sellin R. Follicular and Hurthle cell carcinomas of the thyroid: a comparative study. Am J Surg Pathol 1998; 22:1512-1520.

9. Schlinkert RT, van Heerden JA, Goellner JR, et al. Factors that predict malignant thyroid lesions when fine-needle aspiration is "suspicious for follicular neoplasm”. Mayo Clin Proc 1997;72:913-916. 


\section{PU ET AL.}

10. Tuttle RM, Lemar H, Burch HB. Clinical features associated with an increased risk of thyroid malignancy in patients with follicular neoplasia by fine-needle aspiration. Thyroid 1998;8:377-383.

11. Raber W, Kaserer K, Niederle B, et al. Risk factors for malignancy of thyroid nodules initially identified as follicular neoplasia by fineneedle aspiration: results of a prospective study of one hundred twenty patients. Thyroid 2000;10:709-712.

12. Greaves TS, Olvera M, Florentine BD, et al. Follicular lesions of thyroid: a 5-year fine-needle aspiration experience. Cancer 2000;90:335341.

13. Kim ES, Nam-Goong IS, Gong G, et al. Postoperative findings and risk for malignancy in thyroid nodules with cytological diagnosis of the socalled "follicular neoplasm". Korean J Intern Med 2003;18: 94-97.
14. Sclabas GM, Staerkel GA, Shapiro SE, et al. Fine-needle aspiration of the thyroid and correlation with histopathology in a contemporary series of 240 patients. Am J Surg 2003;186:702-709; discussion 709-710.

15. Guidelines of the Papanicolaou Society of Cytopathology for the examination of fine-needle aspiration specimens from thyroid nodules. The Papanicolaou Society of Cytopathology Task Force on Standards of Practice. Diagn Cytopathol 1996;15:84-89.

16. Baloch ZW, Fleisher S, LiVolsi VA, et al. Diagnosis of "follicular neoplasm": a gray zone in thyroid fine-needle aspiration cytology. Diagn Cytopathol 2002;26:41-44.

17. Davis NL, Gordon M, Germann E, et al. Clinical parameters predictive of malignancy of thyroid follicular neoplasms. Am J Surg 1991;161: $567-569$ 